\title{
SUSCEPTIBILITY OF MICRO-ORGANISMS TO CHLORAMPHENICOL (CHLOROMYCETIN) ${ }^{1,2}$
}

\author{
By I. W. MCLEAN, JR., J. L. SCHWAB, A. B. HILLEGAS, AND A. S. SCHLINGMAN 3
}

(From the Research Laboratories, Parke, Davis \& Co., Detroit 32, Mich.)

The antibiotic, chloramphenicol, produced by Streptomyces venezuelae, was originally reported by Ehrlich et al. $(1,2)$ and independently described by Carter, Gottlieb et al. $(3,4)$. Since these original reports the active principle has been chemically isolated and characterized $(5,6)$, synthesized (7) and is at present commercially available for clinical use.

Originally (1) activity was described against several Gram-negative bacteria, in particular Shigella sonnei, and against Rickettsia prowazekii in chick embryos. Smadel et al. (8-11) have notably extended this spectrum to include other rickettsial agents as well as two viruses, lymphogranuloma venereum and psittacosis. Smith $\boldsymbol{e t}$ al. (12) reported that chloramphenicol was active, under the conditions tested, against Gram-negative bacteria, $R$. prowazekii and Borellia recurrentis; moderately active against Gram-positive bacteria and Mycobacterium tuberculosis; and inactive in the concentrations used against yeasts, filamentous fungi, protozoa and certain viruses. Youmans $e t$ al. (13) tested strains of virulent human-type $M$. tuberculosis in vitro and concluded that chloramphenicol was only moderately active when compared with streptomycin or para-amino salicylic acid.

Clinically, confirmation of laboratory activity has been obtained for certain of the rickettsial diseases (14-18), typhoid fever (19), brucellosis (20) and in urinary infections caused by certain Gram-negative bacteria (21). With the drug now available in quantity for clinical trial, it seems opportune at this time to collect the known informa-

1 Presented at the Second National Symposium on Recent Advances in Antibiotics Research held in Washington, D. C., April 11-12, 1949, under the auspices of the Antibiotics Study Section, National Institutes of Health, Public Health Service, Federal Security Agency.

2 Parke, Davis \& Company trade-name.

Collaborators: F. A. Miller, J. S. Markey, A. Pucheu, M. C. Galbraith, M. E. Doles, M. F. Sheehan, G. Coffey, J. M. McCracken, B. D. Moore, II, M. C. Manning. Research Laboratories, Parke, Davis \& Company. tion with respect to the microbiological spectrum of this new chemotherapeutic agent.

\section{METHODS}

\section{Viruses and Rickettsiae}

Borreliota: Vaccinia virus (New York Public Health strain from Dr. S. K. Muckenfus, New York City Dept. of Public Health, N. Y., egg-adapted) was tested as a representative of this group of viruses. Two types of tests in chick embryos were used. The first consisted of mixing the chloramphenicol with the virus and inoculating $0.1 \mathrm{ml}$. of the mixture onto the chorioallantoic membrane of 10-day chick embryos. After four days incubation at $35^{\circ} \mathrm{C}$. the membranes were examined and compared with controls for reduction in pock formation. The second method consisted of inoculating groups of treated, 10-day chick embryos via the yolk sac with a lethal dose of virus and repeating treatment on the following day by the same route. The eggs were candled twice daily and delay in death time and seven-day survivors recorded.

Erro: St. Louis encephalitis virus (egg-adapted from Dr. Carl Duffy, Wayne University, Detroit, Michigan, and a mouse-adapted laboratory strain) was tested to represent the group of virus encephalitides. Tests were conducted in embryonated eggs and mice. For the egg test, nine-day embryos were infected via the allantoic sac and treated 30 minutes before infection and on the 1st day after infection. The eggs were candled twice daily and time of death and seven-day survivors noted. In the mouse test, groups of 10 to $12 \mathrm{Gm}$. white Swiss mice were infected intracerebrally and treated intraperitoneally before infection and twice daily thereafter for 10 doses. Mean death time was compared with that of a control group.

Formido: The National Institutes of Health mouseadapted strain of rabies virus was used, as well as a variant line of this strain adapted to the intramuscular route of infection. For the former, mice were injected intracerebrally with a lethal dose and daily treatments given intraperitoneally for four days. A similar procedure was used with the intramuscular strain except for the different route of virus administration. Time of paralysis and death of the treated mice was noted for comparison with control groups.

Legio: The anti-poliomyelitis virus tests were conducted by Drs. J. L. Melnick and F. R. Corria (22) of the Yale University School of Medicine, New Haven, Conn. The Y-S.K. and Lansing strains (mouse-adapted) were used as well as Theiler's intestinal virus of mice. 
Rabula: The egg-adapted mumps virus tested was obtained from Dr. J. E. Smadel of the Army Medical School, Washington, D. C. The tests were conducted in seven-day embryonated eggs infected via the allantoic sac. Treatments were given 30 minutes before infection and on the second day. Allantoic fluid was harvested from each egg after seven-days incubation and tested for virus multiplication by the addition of washed chicken red blood cells. In addition a hemagglutination titration was performed on an aliquot pool of allantoic fluid from the groups of eggs. An untreated control group was included in the test for comparison of the number of positive eggs and level of group hemagglutinin titer.

Tarpeia: Tests against three strains of type $A$, seven strains of type $A^{\prime}$ and two strains of type $B$ influenza virus have been conducted in 11-day chick embryos. The virus was inoculated into the allantoic sac and treatments given by the same route 30 minutes before infection and 60 minutes later. After 40 hours incubation at $35^{\circ} \mathrm{C}$. the presence of infection in each egg and the hemagglutination titer of an aliquot pool of allantoic fluid were determined for each group as described above for mumps virus. Considerable work has been done using the mouseadapted PR8 strain of type A in mice. In general, groups of 12 to $14 \mathrm{Gm}$. white Swiss mice were infected intranasally and treated by various routes and at different times with chloramphenicol. In the test reported here treatment was given intraperitoneally for seven days starting at the time of infection.

One inconclusive test has been made with the Green strain of distemper virus (commercial distemperoid, ferretadapted) in ferrets. Two ferrets were infected intranasally and one was treated intraperitoneally at the time of infection and daily thereafter for three days. Severity of illness and time of development of fever were noted. The animals were sacrificed at the height of the disease; hence duration of illness could not be determined.

Tortor: Newcastle disease virus (California 14 from Dr. L. T. Giltner, Bureau of Animal Industry, Washington, D. C.) was tested in 11-day embryonated eggs and white Leghorn pullets. The test in eggs was conducted in the same manner as the influenza virus tests described above except that infection was determined by death or survival of the embryo. Comparisons of the average time of death of treated and untreated groups were made. In chickens the virus was administered intramuscularly and treatments were given approximately every 12 hours for four doses starting at the time of infection. Time of the appearance of symptoms and number of survivors compared with a control group were noted.

The chick bronchitis virus (obtained from Dr. C. $\mathbf{H}$. Cunningham, Michigan State College, East Lansing, Michigan) was tested in chick embryos as described above for Newcastle disease.

Laryngo-tracheitis virus (commercial vaccine strain) was tested on the membrane of chick embryos using the technique described for vaccinia virus.

Rickettsia: Considerable work has been done with epidemic typhus ( $R$. prowazekii, Breinl strain) in chick embryos. In the work reported here, groups of six-day chick embryos were infected via the yolk sac and treated once on the third day after infection by the same route. Average time of death and number of survivors were compared with an untreated control group.

The results with murine typhus ( $R$. typhi), scrub typhus ( $R$. tsutsugamushi) and rickettsialpox ( $R$. akari) were taken from the work of Smadel et al. (8-11). Using the method described above, their findings for murine typhus in eggs have been confirmed in this laboratory.

Dermacentroxenus, Coxiella and Miyagawanella: The reports of Smadel et al. (8-11) are included in the table for Rocky Mountain spotted fever (D. rickettsi), $Q$ fever (C. burnetii), lymphogranuloma venereum (M. lymphogranulomatis) and psittacosis (M. psittacii).

\section{Bacteria}

The bacterial species reported here were tested for their susceptibility to chloramphenicol by inoculating a specified dilution or quantity of liquid culture into media containing varying concentrations of the drug, the end point being taken as the lowest concentration which caused complete inhibition of growth for 18 hours. Except as indicated the original transplants for preparation of suspensions were made from cultures dried from the frozen state.

The aerobes were tested by adding $1.0 \mathrm{ml}$. of a standardized dilution of a tryptose broth culture to $1.0 \mathrm{ml}$. quantities of tryptose broth containing varying concentration of chloramphenicol. The final concentrations used varied from 0.1 to $200 \mu \mathrm{g} / \mathrm{ml}$. The tubes were incubated for 18 hours and the results recorded as "growth" or "no growth."

It was necessary to utilize special media for some of the more fastidious organisms. However, the same technique was used, replacing the tryptose broth with the special medium required. Brucella abortus was grown under 10 per cent $\mathrm{CO}_{2}$ for 48 hours (No. 1335).

The anaerobes were tested by adding varying concentrations (ranging from 0.1 to $500 \mu \mathrm{g} / \mathrm{ml}$.) of chloramphenicol to melted agar medium cooled to $40^{\circ} \mathrm{C}$. To each tube, $0.1 \mathrm{ml}$. of a 24-hour liquid culture of the organism to be tested was added. The tubes were then shaken thoroughly and allowed to gel. Growth was recorded as positive or negative after 24 hours incubation.

Beef infusion agar ( 2 per cent) containing 2 per cent glucose ( $\mathrm{pH}$ 8.2) was used for Clostridium perfringens, novyi and septicum. Cl. feseri was tested in 2 per cent liver infusion agar ( $\mathrm{pH}$ 8.2) and the anaerobic agar recommended by Records and Vawter (23) was utilized for $\mathrm{Cl}$. hemolyticum. Cl. tetani was grown in Taylor medium containing 0.5 per cent agar and incubated in an atmosphere of hydrogen and carbon dioxide.

\section{Fungi}

In determining the susceptibility of fungi to chloramphenicol, a broth dilution method was employed. Serial dilutions of the drug in broth were dispensed in $1 \mathrm{ml}$. amounts in Wassermann tubes and to each tube was added 
$1 \mathrm{ml}$. of inoculum in broth. The tubes were incubated at $30^{\circ} \mathrm{C}$. for one to seven days, depending upon the species, and then observed for inhibition of growth. The minimum concentration of the drug causing complete inhibition of growth was taken as the end point.

Modified Sabouraud's broth (dextrose 2 per cent, neo peptone 1 per cent) was used in preparing the dilutions of the drug and the fungus suspensions. In the case of the actinomycetes, Brain-Heart Infusion Broth (Difco) was employed.

Three types of inocula were used. 1) Spore suspension: In heavily sporulating species suspensions were prepared from either agar slant or agar plate cultures of the fungus. In the case of agar slants, broth was added to the tube, the fungus stirred up with an inoculating needle and the tube then shaken. When using agar plate cultures the mycelium was scraped from the surface of the plate, placed in broth and shaken. The suspensions were then filtered through cotton and the spores counted on the hemocytometer. 2) Spores and mycelial fragments: Species having limited sporulation were removed from the surface of the culture medium, emulsified with broth either in a Waring Blender, by shaking with glass beads or by grinding in a mortar. The suspension was filtered through a loose cotton mat and in some instances the number of fragments and spores per ml. was determined by means of the hemocytometer. 3) Mycelial suspension: The mycelium of non-sporulating species was

TABLE I

Viruses and rickettsiae

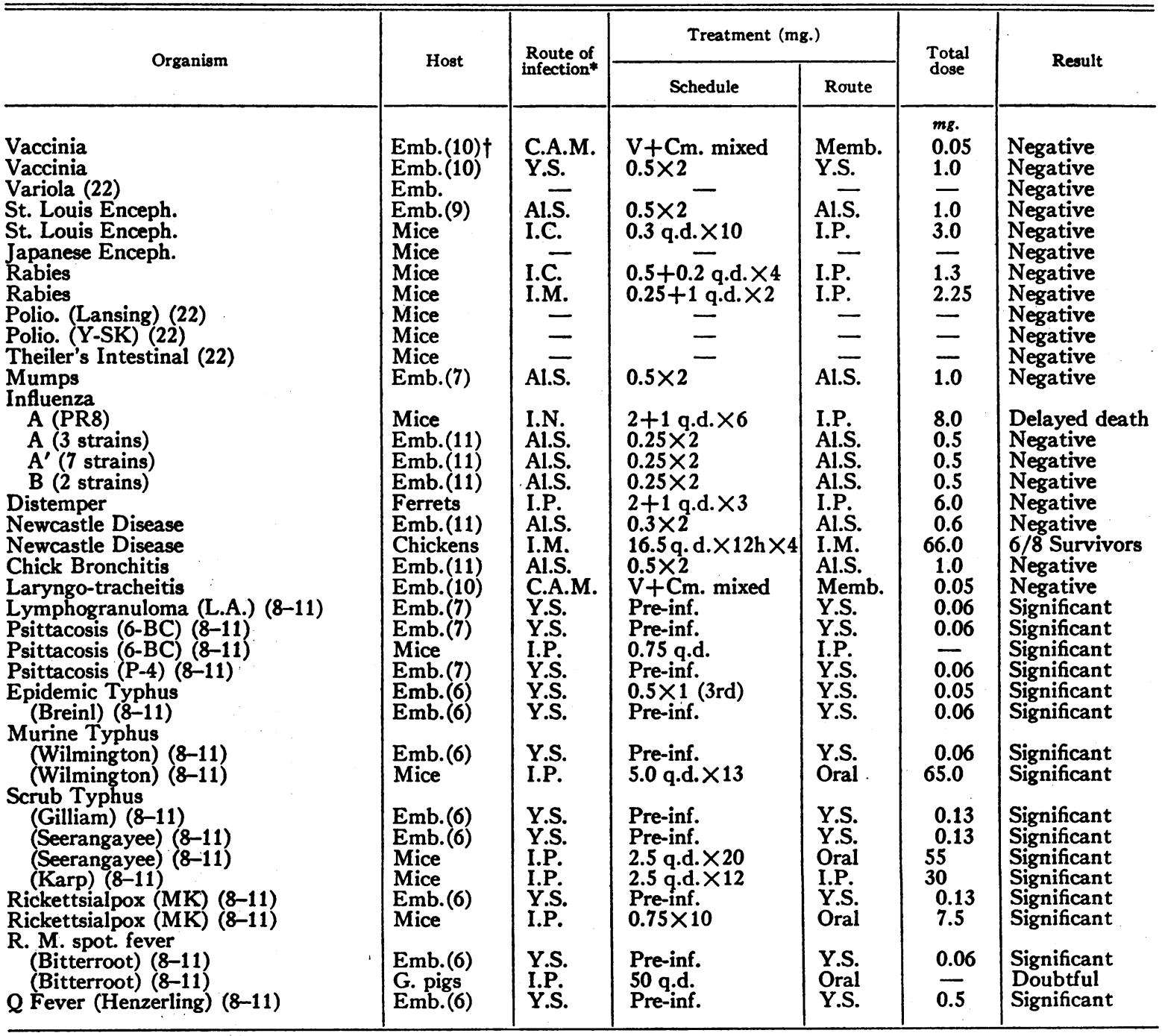

* C.A.M. = chorioallantoic membrane; Y.S. = yolk sac; Al.S. = allantoic sac; I.C. = intracerebral; I.M. = intramuscular; I.N. = intranasal; I.P. = intraperitoneal.

$\dagger$ Emb.(10) = chick embryos and (age of incubation). 
TABLE II

Bacteria

\begin{tabular}{|c|c|c|c|c|}
\hline Organism & $\begin{array}{l}\text { P-D Culture } \\
\text { Bureau No. }\end{array}$ & Source & Inoculum & $\begin{array}{l}\text { Inhibiting } \\
\text { conc. }\end{array}$ \\
\hline 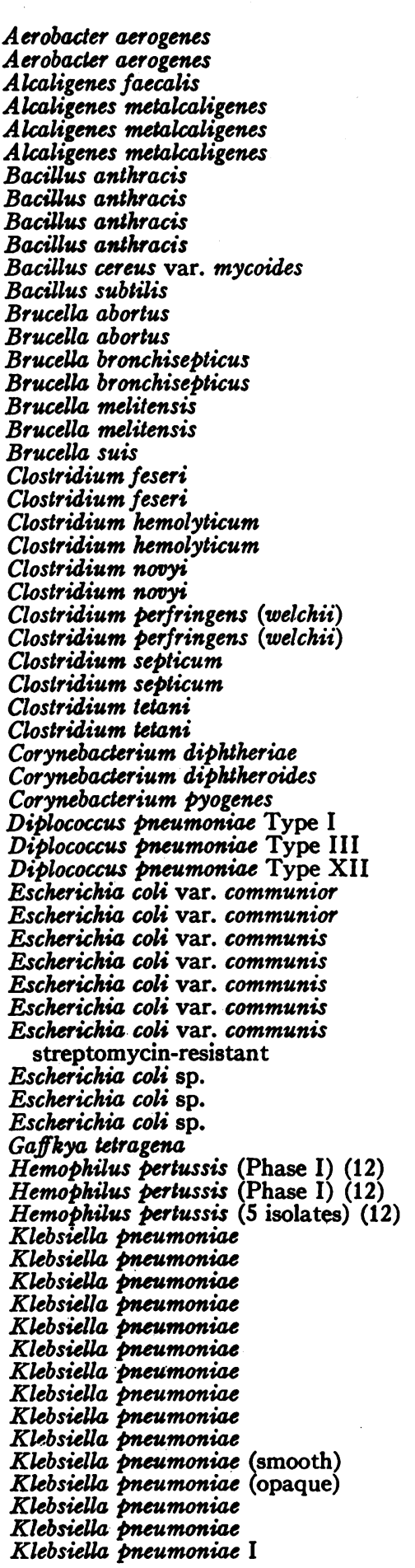 & 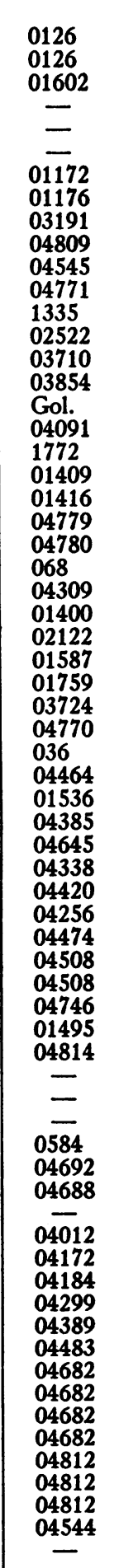 & 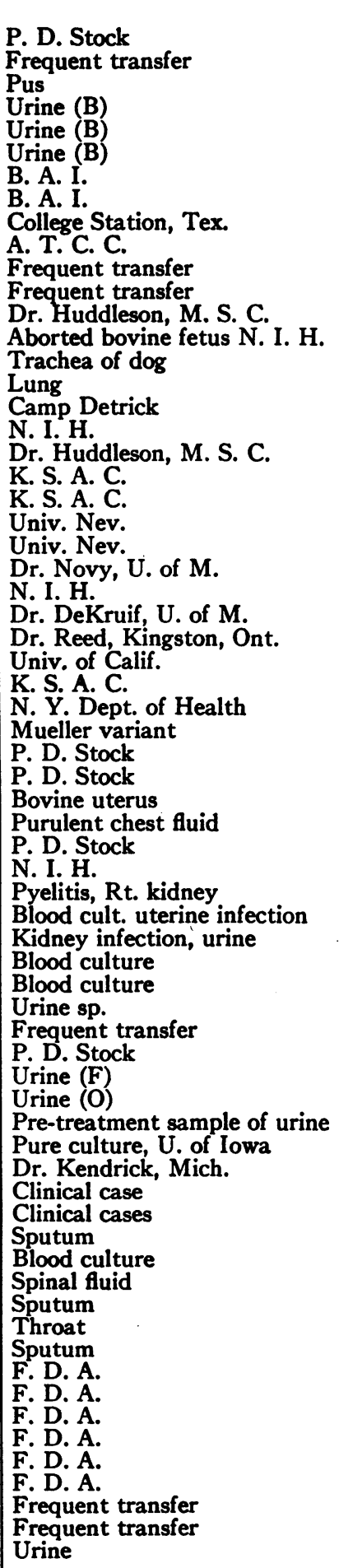 & $\begin{array}{l}1-20,000,000 \\
1-10,000,000 \\
1-10,000,000 \\
1-10,000,000 \\
1-10,000,000 \\
1-10,000,000 \\
1: 20 \\
1: 20 \\
1: 10 \\
1: 20 \\
1-10,000,000 \\
1-10,000,000 \\
1: 20 \\
1: 20 \\
1: 20 \\
1: 20 \\
1: 20 \\
1: 20 \\
1: 20 \\
0.1 \text { cc. undiluted } \\
0.1 \text { cc. undiluted } \\
0.1 \text { cc. undiluted } \\
0.1 \text { cc. undiluted } \\
0.1 \text { cc. undiluted } \\
0.1 \text { cc. undiluted } \\
0.1 \text { cc. undiluted } \\
0.1 \text { cc. undiluted } \\
0.1 \text { cc. undiluted } \\
0.1 \text { cc. undiluted } \\
0.1 \text { cc. undiluted } \\
0.1 \text { cc. undiluted } \\
1-10,000,000 \\
1-10,000,000 \\
1: 10 \\
1-10,000,000 \\
1-10,000,000 \\
1-5,000,000 \\
1-20,000,000 \\
1-20,000,000 \\
1-10,000,000 \\
1-10,000,000 \\
1-20,000,000 \\
1-20,000,000 \\
1-10,000,000 \\
1-10,000,000 \\
1-10,000,000 \\
1-10,000,000 \\
1-10,000,000 \\
1-10,000,000 \\
104 \text { organisms } \\
104 \text { organisms } \\
104 \text { organisms } \\
1-10,000,000 \\
1-10,000,000 \\
1-10,000,000 \\
1-10,000,000 \\
1-10,000,000 \\
1-10,000,000 \\
1-500,000 \\
1-1,000,000 \\
1-10,000,000 \\
1-20,000,000 \\
1-20,000,000 \\
1-10,000,000 \\
1-20,000,000 \\
1-10,000,000 \\
1-10,000,000 \\
10\end{array}$ & 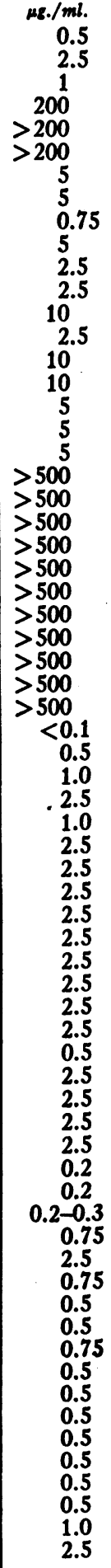 \\
\hline
\end{tabular}


TABLE II-Continued

\begin{tabular}{|c|c|c|c|c|}
\hline Organism & $\begin{array}{l}\text { P-D Culture } \\
\text { Bureau No. }\end{array}$ & Source & Inoculum & $\begin{array}{l}\text { Inhibiting } \\
\text { conc. }\end{array}$ \\
\hline $\begin{array}{l}\text { Klebsiella pneumoniae II } \\
\text { Malleomyces mallei } \\
\text { Micrococcus citreus } \\
\text { Micrococcus citreus } \\
\text { Micrococcus pyogenes var. albus } \\
\text { Micrococcus pyogenes var. albus } \\
\text { Micrococcus pyogenes var. albus } \\
\text { Micrococcus pyogenes var. albus } \\
\text { Micrococcus pyogenes var. aureus } \\
\text { Micrococcus pyogenes var. aureus } \\
\text { Micrococcus pyogenes var. aureus } \\
\text { Micrococcus pyogenes var. aureus } \\
\text { Micrococcus pyogenes var. aureus } \\
\text { Micrococcus pyogenes var. aureus } \\
\text { Micrococcus pyogenes var. aureus } \\
\text { Micrococcus pyogenes var. aureus } \\
\text { Micrococcus pyogenes var. aureus } \\
\text { Micrococcus pyogenes var. aureus } \\
\text { Micrococcus pyogenes var. aureus } \\
\text { Micrococcus pyogenes var. aureus } \\
\text { Micrococcus pyogenes var. aureus } \\
\text { Mycobacterium phlei } \\
\text { Mycobacterium.tuberculosis (13) } \\
\text { (human virulent, } 12 \text { strains) } \\
\text { Neisseria catarrhalis } \\
\text { Neisseria catarrhalis } \\
\text { Neisseria catarrhalis } \\
\text { Neisseria meningitidis } \\
\text { Pasteurella avicida } \\
\text { Pasteurella avicida } \\
\text { Pasteurella avicida } \\
\text { Pasteurella bollingeri } \\
\text { Pasteurella bollingeri } \\
\text { Pasteurella bubalseptica } \\
\text { Pasteurella cuniculicida } \\
\text { Pasteurella oviseptica } \\
\text { Proteus vulgaris } \\
\text { Proteus vulgaris } \\
\text { Proteus vulgaris } \\
\text { Proteus vulgaris } \\
\text { Proteus vulgaris } \\
\text { Proteus vulgaris } \\
\text { Proteus species No. } 1 \\
\text { Proteus species No. } 2 \\
\text { Proteus species No. } 3 \\
\text { Proteus species No. } 4 \\
\text { Pseudomonas aeruginosa } \\
\text { Pseudomonas aeruginosa } \\
\text { Pseudomonas aeruginosa } \\
\text { Pseudomonas aeruginosa } \\
\text { Pseudomonas aeruginosa } \\
\text { Pseudomonas aeruginosa } \\
\text { Pseudomonas aeruginosa } \\
\text { Pseudomonas aeruginosa } \\
\text { Pseudomonas aeruginosa } \\
\text { Pseudomonas aeruginosa } \\
\text { Pseudomonas aeruginosa } \\
\text { Pseudomonas aeruginosa } \\
\text { Pseudomonas aeruginosa } \\
\text { Pseudomonas aeruginosa } \\
\text { Pseudomonas aeruginosa } \\
\text { Pseudomonas aeruginosa (rough type) } \\
\text { Pseudomonas aeruginosa (smooth type) } \\
\text { Pseudomonas aeruginosa } \\
\text { Pseudomonas aeruginosa } \\
\text { Rhodospirillum rubrum } \\
\text { Salmonella enteritidis } \\
\text { Salmonella enteritidis } \\
\text { Salmonella gallinarum } \\
\text { Py }\end{array}$ & 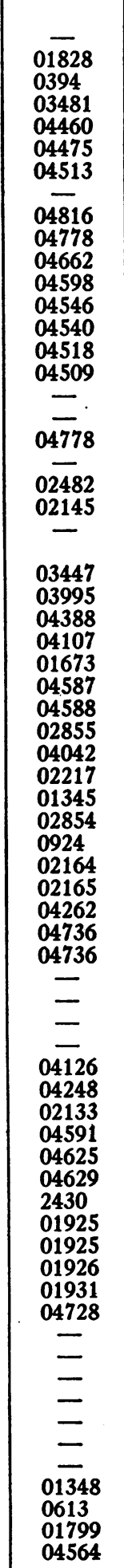 & 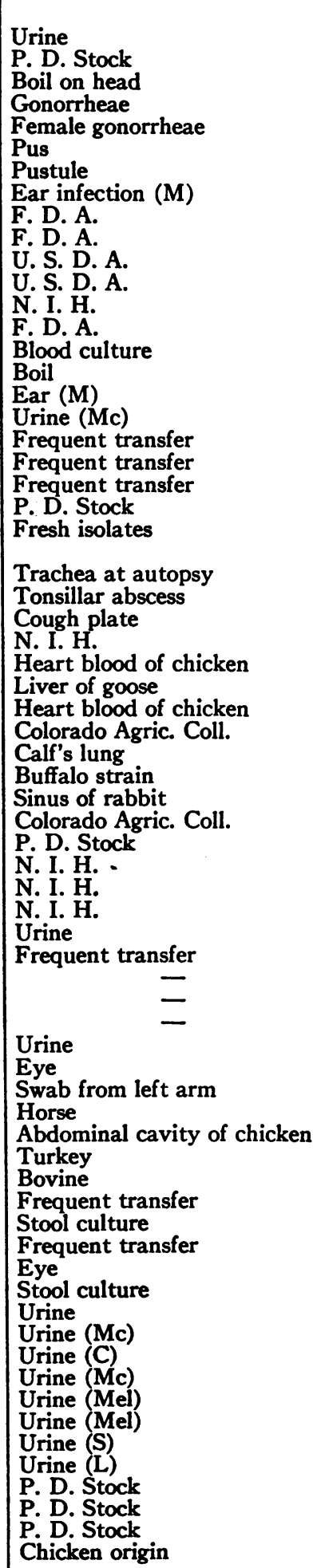 & $\begin{array}{l}1-5,000,000 \\
1: 2000 \\
1-10,000,000 \\
1-10,000,000 \\
1-10,000,000 \\
1-10,000,000 \\
1-10,000,000 \\
1-5,000,000 \\
1-10,000,000 \\
1-10,000,000 \\
1-10,000,000 \\
1-10,000,000 \\
1-10,000,000 \\
1-10,000,000 \\
1-10,000,000 \\
1-10,000,000 \\
1-5,000,000 \\
1-5,000,000 \\
1-10,000,000 \\
1-10,000,000 \\
1-10,000,000 \\
1-10,000,000 \\
\\
1-10,000,000 \\
1-5,000,000 \\
1-5,000,000 \\
1-5,000,000 \\
1: 2000 \\
1: 2000 \\
1: 2000 \\
1: 2000 \\
1: 10 \\
1: 2000 \\
1: 2000 \\
1: 2000 \\
1-10,000,000 \\
1-10,000,000 \\
1-10,000,000 \\
1-10,000,000 \\
1-10,000,000 \\
1-10,000,000 \\
1-10,000,000 \\
1-10,000,000 \\
1-10,000,000 \\
1-10,000,000 \\
1-10,000,000 \\
1-10,000,000 \\
1-10,000,000 \\
1-10,000,000 \\
1-10,000,000 \\
1-10,000,000 \\
1-10,000,000 \\
1-10,000,000 \\
1-10,000,000 \\
1-10,000,000 \\
1-10,000,000 \\
1-10,000,000 \\
1-5,000,000 \\
1-10,000,000 \\
1-10,000,000 \\
1-5,000,000 \\
1-10,000,000 \\
1-10,000,000 \\
1-5,000,000 \\
1-10,000,000 \\
1-10,000,000 \\
1-10,000,000 \\
1-10,000,000\end{array}$ & 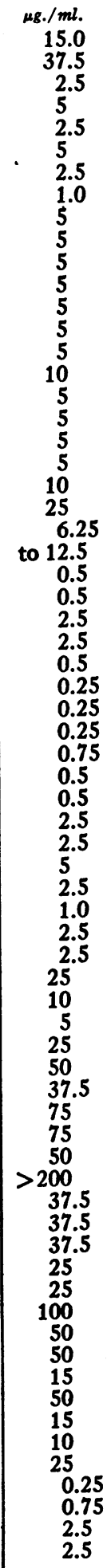 \\
\hline
\end{tabular}


TABLE II-Continued

\begin{tabular}{|c|c|c|c|c|}
\hline Organism & $\begin{array}{l}\text { P-D Culture } \\
\text { Bureau No. }\end{array}$ & Source & Inoculum & $\begin{array}{l}\text { Inhibiting } \\
\text { conc. }\end{array}$ \\
\hline $\begin{array}{l}\text { Salmonella gallinarum } \\
\text { Salmonella paratyphi } \\
\text { Salmonella paratyphi } \\
\text { Salmonella schottmuelleri } \\
\text { Salmonella schottmuelleri } \\
\text { Salmonella schottmuelleri } \\
\text { Salmonella typhimurium } \\
\text { Salmonella typhimurium } \\
\text { Salmonella typhimurium } \\
\text { Salmonella typhosa } \\
\text { Salmonella typhosa } \\
\text { Salmonella typhosa } \\
\text { Salmonella typhosa } \\
\text { Salmonella typhosa } \\
\text { Salmonella typhosa } \\
\text { Salmonella typhosa } \\
\text { Salmonella typhosa } \\
\text { Salmonella typhosa } \\
\text { Salmonella typhosa } \\
\text { Salmonella typhosa } \\
\text { Salmonella typhosa } \\
\text { Salmonella typhosa } \\
\text { Salmonella typhosa } \\
\text { Salmonella typhosa } \\
\text { Salmonella typhosa } \\
\text { Salmonella typhosa } \\
\text { Salmonella typhosa } \\
\text { Salmonella typhosa } \\
\text { Salmonella typhosa } \\
\text { Salmonella typhosa } \\
\text { Salmonella typhosa } \\
\text { Salmonella typhosa } \\
\text { Salmonella typhosa } \\
\text { Salmonella typhosa } \\
\text { Sarcina aurantiaca } \\
\text { Sarcina lutea } \\
\text { Sarcina lutea } \\
\text { Serratia marcescens } \\
\text { Serratia marcescens } \\
\text { Serratia marcescens } \\
\text { Serratia marcescens } \\
\text { Shigella dysenteriae } \\
\text { Shigella dysenteriae } \\
\text { Shigella madampensis } \\
\text { Shigella madampensis } \\
\text { Shigella paradysenteriae } \\
\text { (Bacillus dysenteriae Flexner I) } \\
\text { (Bacillus dysenteriae Flexner II) } \\
\text { (Bacillus dysenteriae Flexner II) } \\
\text { (Bacillus dysenteriae Flexner III) } \\
\text { (Bacillus dysenteriae Flexner VI) } \\
\text { (Bacillus dysenteriae Flexner VII) } \\
\text { (Bacillus dysenteriae Flexner VIII) } \\
\text { (Bacillus dysenteriae var.) } \\
\text { Shigella sonnei } \\
\text { Shigella sonnei } \\
\text { Shigella sonnei } \\
\text { Shigella sonnei } \\
\text { Streptococcus hemolyticus } \\
\text { Streptococcus hemolyticus } \\
\text { Streptococcus hemolyticus } \\
\text { Streptococcus hemolyticus } \\
\text { Streptococcus infrequens } \\
\text { Streptococcus lactis } \\
\text { Streptococcus non-hemolyticus } \\
\text { Streptococcus non-hemolyticus } \\
\text { Vibrio comma } \\
\text { al }\end{array}$ & $\begin{array}{l}04574 \\
01179 \\
02156 \\
01180 \\
01180 \\
01181 \\
03869 \\
0301 \\
04221 \\
04425 \\
04469 \\
04537 \\
04599 \\
04683 \\
04788 \\
04815 \\
02481 \\
- \\
- \\
- \\
- \\
- \\
- \\
- \\
- \\
- \\
- \\
- \\
- \\
- \\
- \\
- \\
- \\
044764 \\
03454 \\
04622 \\
04150 \\
04643\end{array}$ & $\begin{array}{l}\text { Turkey } \\
\text { N. I. H. } \\
\text { N. I. H. } \\
\text { N. I. H. } \\
\text { Frequent transfer } \\
\text { N. I. H. } \\
\text { Rabbit typhoid } 104 \\
\text { Rat } \\
\text { Heart blood of lamb } \\
\text { P. D. Stock } \\
\text { N. I. H. } \\
\text { N. I. H. } \\
\text { N. I. H. } \\
\text { N. T. C. C. } \\
\text { Stool culture } \\
\text { Typhoid carrier } \\
\text { Frequent transfer } \\
\text { Typhoid carrier } \\
\text { Typhoid carrier } \\
\text { Typhoid carrier } \\
\text { Typhoid carrier } \\
\text { Typhoid carrier } \\
\text { Typhoid carrier } \\
\text { Typhoid carrier } \\
\text { Typhoid carrier } \\
\text { Typhoid carrier } \\
\text { Typhoid carrier } \\
\text { Typhoid carrier } \\
\text { Typhoid carrier } \\
\text { Typhoid carrier } \\
\text { Typhoid carrier } \\
\text { Typhoid carrier (M.C.)* } \\
\text { Typhoid carrier (M.C.)† } \\
\text { Typhoid carrier (J.T.)† } \\
\text { P. D. Stock } \\
\text { F. D. A. } \\
\text { Frequent transfer } \\
\text { P. D. Stock } \\
\text { A. T. C. C. } \\
\text { A. T. C. C. } \\
\text { P. D. Stock } \\
\text { P. D. Stock } \\
\text { N. I. H. } \\
\text { A. T. C. C. } \\
\text { A. T. C. C. } \\
\text { A. T. C. C. } \\
\text { P. D. Stock } \\
\text { P. D. Stock } \\
\text { A. T. C. C. } \\
\text { P. D. Stock } \\
\text { P. D. Stock } \\
\text { P. D. Stock } \\
\text { P. D. Stock } \\
\text { P. D. Stock } \\
\text { P. D. Stock } \\
\text { P. D. Stock } \\
\text { Frequent transfer } \\
\text { Abscess } \\
\text { P. D. Stock } \\
\text { Group A } \\
\text { Frequent transfer } \\
\text { Frequent transfer } \\
\text { Pasteurized milk } \\
\text { P. D. Stock } \\
\text { Extracted tooth } \\
\text { N. I. H. } \\
\text { Ty } \\
\text { Ty }\end{array}$ & $\begin{array}{l}1-10,000,000 \\
1-10,000,000 \\
1-10,000,000 \\
1-10,000,000 \\
1-10,000,000 \\
1-10,000,000 \\
1-10,000,000 \\
1-10,000,000 \\
1-10,000,000 \\
1-10,000,000 \\
1-10,000,000 \\
1-10,000,000 \\
1-10,000,000 \\
1-10,000,000 \\
1-10,000,000 \\
1-10,000,000 \\
1-10,000,000 \\
1-10,000,000 \\
1-10,000,000 \\
1-10,000,000 \\
1-10,000,000 \\
1-10,000,000 \\
1-10,000,000 \\
1-10,000,000 \\
1-10,000,000 \\
1-10,000,000 \\
1-10,000,000 \\
1-10,000,000 \\
1-10,000,000 \\
1-10,000,000 \\
1-10,000,000 \\
1-10,000,000 \\
1-10,000,000 \\
1-10,000,000 \\
1-5,000,000 \\
1-5,000,000 \\
1-10,000,000 \\
1-5,000,000 \\
1-10,000,000 \\
1-10,000,000 \\
1-10,000,000 \\
1-10,000,000 \\
1-10,000,000 \\
1-10,000,000 \\
1-10,000,000 \\
1-10,000,000 \\
1-10,000,000 \\
1-10,000,000 \\
1-10,000,000 \\
1-10,000,000 \\
1-10,000,000 \\
1-10,000,000 \\
1-10,000,000 \\
1-10,000,000 \\
1-10,000,000 \\
1-10,000,000 \\
1-10,000,000 \\
1-5,000,000 \\
1-5,000,000 \\
1-5,000,000 \\
1-5,000,000 \\
1-5,000,000 \\
1-5,000,000 \\
1-10,000,000 \\
1-5,000,000 \\
1-2000\end{array}$ & 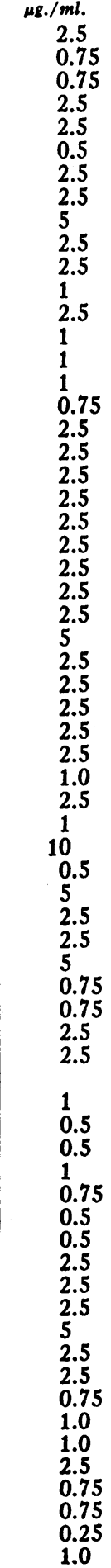 \\
\hline
\end{tabular}

* Pre-treatment. † After treatment. 
processed in the manner described for preparing the suspension of spores and mycelial fragments.

All of the fungi were tested first in a series of dilutions ranging from 50 to $1000 \mu \mathrm{g} / \mathrm{ml}$. of the drug. The actinomycetes, Actinomyces bovis and Nocardia asteroides, were retested at lower concentrations and four species, Trichophyton interdigitale, $T$. mentagrophytes, $T$. rubrum and Microsporum canis, at higher concentrations (up to 2500 $\mu \mathrm{g} / \mathrm{ml}$.) of chloramphenicol. In order to keep the drug in solution at the higher concentrations $1.8 \mathrm{ml}$. of the drug-broth mixture and $0.2 \mathrm{ml}$. of inoculum were used in each tube instead of $1 \mathrm{ml}$. of each as in the lower concentration series.

\section{Spirochetes and Protozoa}

The authors are indebted to Dr. Paul E. Thompson (24) and Dr. O. M. Gruhzit (25) of the Parke, Davis Research Laboratories for the data included in this section. In Vitro Tests: Endamoeba histolytica (University of Chicago strain, with mixed bacterial flora) was tested in two media, Egg-Locke diphasic and Balamuth (essentially protein free). Various concentrations of chloramphenicol were incorporated in these media and amebicidal activity determined by microscopic examination for motility after 24 hours at $37^{\circ} \mathrm{C}$.

Borrelia novyi and recurrentis and Trichomonas foetus

TABLE III

Fungi

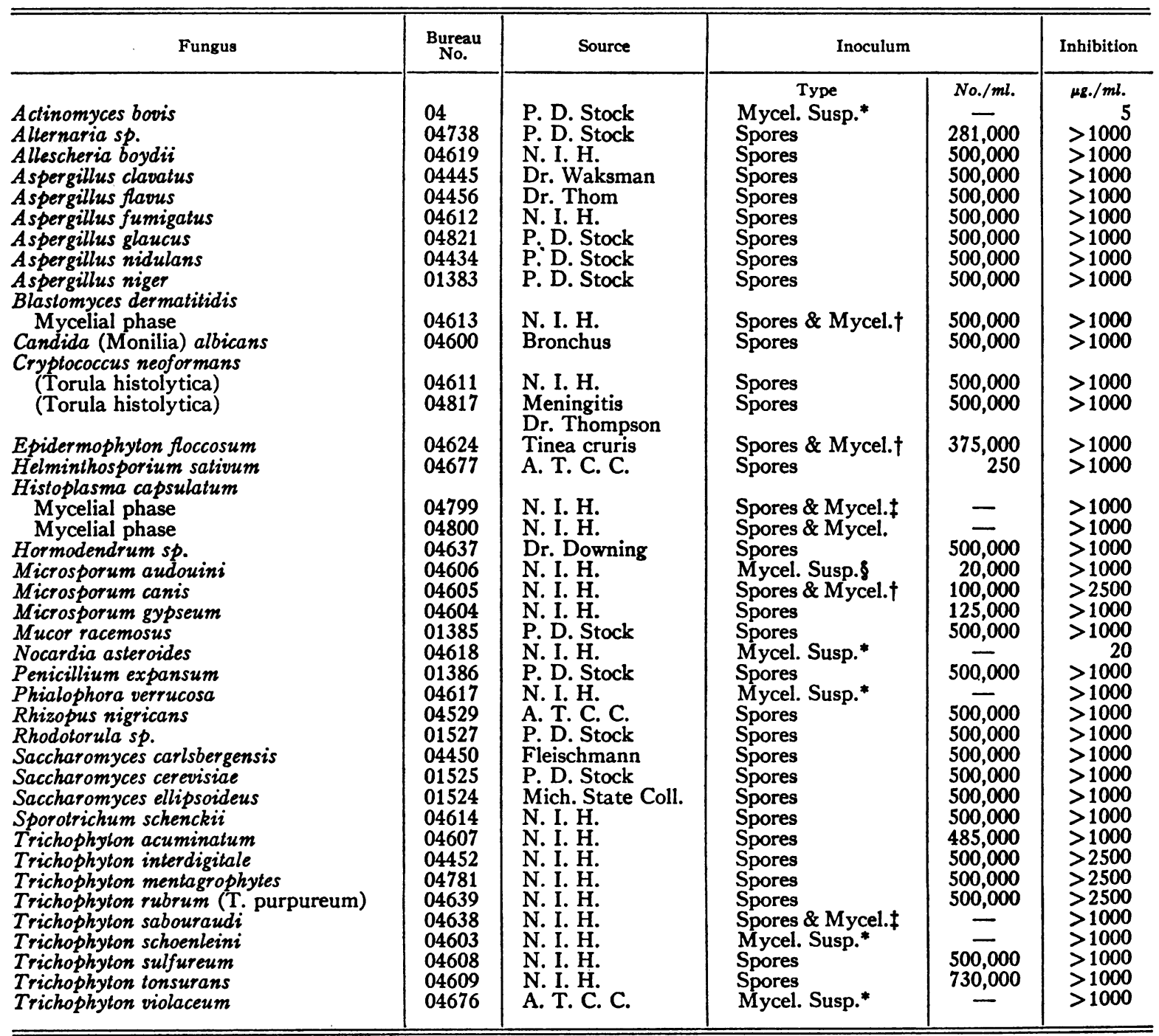

* Suspension of emulsified mycelial material. The number of fragments was not determined.

$\dagger$ Both spores and mycelial fragments are included in the count.

$\ddagger$ Light suspension of spores and mycelial fragments.

Mycelial fragments counted. 
were suspended in a suitable menstruum containing varying concentrations of chloramphenicol, as indicated in Table IV, and observed for immobilization. Observations were made after two hours in the case of the Borreliae and after seven hours exposure for trichomonas.

In Vivo Tests: Plasmodium lophurae was tested in ducks (intraperitoneal treatment) and chicks (oral treatment) and the results compared with the activity of quinine.

Rats and dogs were used to evaluate the activity of chloramphenicol against $E$. histolytica in vivo. Treatment was given orally and results expressed as number of animals cleared of infection or degree of suppression of infection.

$B$. novyi was tested in groups of mice treated by either the intraperitoneal or oral route. Results are expressed as percentage suppression of spirochetemia.

Rabbits infected with Treponema pallidum were treated with various concentrations of chloramphenicol twice daily for eight days. Rate of disappearance of treponemes and degree of healing of lesions under treatment were noted. The animals were held to check for relapses in the dosages at which healing under treatment was noted.

\section{RESULTS}

Tables I to IV summarize the results obtained with the different micro-organisms that have been tested in the laboratory. Wherever possible the minimal inhibiting concentration of chloramphenicol for the strain, under the conditions tested, is given. Otherwise, the maximum concentration tested or the dose administered, in the case of in vivo tests, is entered. The identifying numbers given for the bacteria and fungi are those assigned by the Parke, Davis and Company culture bureau, where a complete history of the strain is available. Strains without assigned numbers are for the most part recent isolates from clinical cases prior to or during treatment with chloramphenicol.

\section{The Emergence of Microbial Resistance}

With the finding of considerable variation in susceptibility to chloramphenicol of different cultural lines of certain bacterial species, it became

TABLE IV

Spirochetes and protozoa

\begin{tabular}{|c|c|c|c|c|}
\hline \multicolumn{5}{|c|}{ In vilro tests } \\
\hline Organism & Medium or menstruum & Time & Conc. & Result \\
\hline $\begin{array}{l}\text { Endamoeba histolytica * } \\
\text { Endamoeba histolytica * } \\
\text { Borrelia novyi } \\
\text { Borrelia recurrentis } \\
\text { Trichomonas foetus } \\
\text { Pelomyxa carolinensis } \\
\text { Tetrahymena geleii }\end{array}$ & $\begin{array}{l}\text { L. E. L. } \\
\text { Balamuth } \\
50 \% \text { horse serum } \\
2.5 \% \text { rat serum } \\
0.7 \% \text { sodium chloride } \\
\text { Pace \& Kimura buffer } \\
2 \% \text { proteose peptone }\end{array}$ & $\begin{array}{r}24 \text { hours } \\
24 \text { hours } \\
2 \text { hours } \\
2 \text { hours } \\
7 \text { hours } \\
48 \text { hours } \\
48 \text { hours }\end{array}$ & $\begin{array}{c}\mu g . / \mathrm{ml} . \\
1000 \\
250 \\
10 \text { to } 50 \\
2.5 \\
2000 \\
2500 \\
2500\end{array}$ & $\begin{array}{l}\text { Negative } \\
\text { Significant } \\
\text { Immobilization } \\
\text { Immobilization } \\
\text { Negative } \\
\text { Negative } \\
\text { Negative }\end{array}$ \\
\hline
\end{tabular}

\begin{tabular}{|c|c|c|c|c|c|}
\hline \multicolumn{6}{|c|}{ In vivo tests } \\
\hline \multirow{2}{*}{ Organism } & \multirow{2}{*}{ Host } & \multicolumn{3}{|c|}{ Treatment } & \multirow{2}{*}{ Result } \\
\hline & & Dose & Schedule & Route & \\
\hline $\begin{array}{l}\text { Plasmodium lophurae } \\
\text { Plasmodium lophurae } \\
\text { Endamoeba histolytica } \\
\text { Endamoeba histolytica } \\
\text { Borrelia novyi } \\
\text { Borrelia novyi } \\
\text { Treponema pallidum } \\
\text { Treponema pallidum }\end{array}$ & $\begin{array}{l}\text { Ducks } \\
\text { Chicks } \\
\text { Rats } \\
\text { Dogs } \\
\text { Mice } \\
\text { Mice } \\
\text { Rabbits } \\
\text { Rabbits }\end{array}$ & $\begin{array}{c}\text { mg./kg./day } \\
200 \\
537 \\
583-868 \\
200 \\
14.9 \\
7.6 \\
25 \\
50-100\end{array}$ & 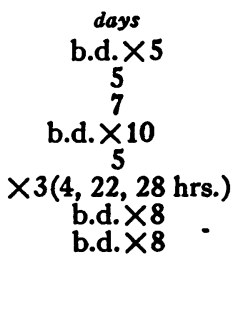 & $\begin{array}{l}\text { I.P. } \\
\text { Diet } \\
\text { Diet } \\
\text { Oral } \\
\text { Diet } \\
\text { I.P. } \\
\text { I.M. } \\
\text { I.M. }\end{array}$ & $\begin{array}{l}\mathrm{Q} \|=<0.05 \\
\mathrm{Q}=<0.1 \\
\mathbf{9} / \mathbf{1 4} \text { cleared } \\
\text { Suppression } \\
50 \% \text { Suppression } \\
50 \% \text { Suppression } \\
\text { Negative } \\
\text { Spirochetes and lesions } \\
\text { cleared 4-5 days, re- } \\
\text { lapsed }\end{array}$ \\
\hline
\end{tabular}

* Thompson, P. E., Research Laboratories of Parke, Davis \& Company, personal communication and (12).

t Reutner, T. F., Research Laboratories of Parke, Davis \& Company and (12).

$\$$ Pace, D. M., and Russell, D., quoted (12).

Gruhzit, O. M., personal communication, Research Laboratories of Parke, Davis \& Company.

II Quinine equivalent. 
TABLE V

Induced resistance to chloramphenicol in bacteria

\begin{tabular}{|c|c|c|c|c|}
\hline \multirow{2}{*}{ Organism } & \multirow{2}{*}{$\begin{array}{c}\text { P-D } \\
\text { Culture } \\
\text { Bureau No. }\end{array}$} & \multirow{2}{*}{$\begin{array}{l}\text { Transfers on } \\
\text { chloramphenicol } \\
\text { containing } \\
\text { medium }\end{array}$} & \multicolumn{2}{|c|}{$\begin{array}{l}\text { Inhibiting conc. chloramphenicol } \\
\mu \mathrm{g} . / \mathrm{ml} \text {. }\end{array}$} \\
\hline & & & Original & Final \\
\hline $\begin{array}{l}\text { Salmonella typhosa } \\
\text { A erobacter aerogenes } \\
\text { Escherichia coli } \\
\text { Proteus (sp.) } \\
\text { Pseudomonas aeruginosa } \\
\text { Klebsiella pneumoniae } \\
\text { Alcaligenes metalcaligenes }\end{array}$ & $\begin{array}{c}\text { clinical case } \\
0126 \\
01495 \\
04736 \\
01925 \\
04544 \\
\text { clinical case }\end{array}$ & $\begin{array}{r}12 \\
12 \\
12 \\
8 \\
8 \\
12 \\
7\end{array}$ & $\begin{array}{r}10 \\
5 \\
10 \\
500 \\
1250 \\
5 \\
1250\end{array}$ & $\begin{array}{r}250 \\
50 \\
500 \\
>4000 \\
>4000 \\
15 \\
2250\end{array}$ \\
\hline
\end{tabular}

of interest to determine whether increased resistance to chloramphenicol could be induced by laboratory manipulation. Our early observations indicate that certain species of micro-organisms will develop resistance to chloramphenicol when sub-inoculated on media containing increasing concentrations of the drug. The bacterial species tested include Salmonella typhosa, Aerobacter aerogenes, Escherichia coli, Proteus sp., Pseudomonas aeruginosa, Klebsiella pneumoniae, and Alcaligenes metalcaligenes.

The test was carried out by sub-inoculating 0.1 $\mathrm{ml}$. of the various cultures into Brain-Heart Infusion Broth (Difco) with 10 per cent horse serum added and containing various concentrations of chloramphenicol. The cultures were incubated for 72-96 hours. From the tube containing the highest concentration of chloramphenicol, in which good growth was obtained, $0.1 \mathrm{ml}$. was sub-inoculated into $2.0 \mathrm{ml}$. of medium containing the same and higher concentrations of the drug. In Table $\mathrm{V}$ the preliminary results of this study are indicated. It can be seen that the susceptibility of these organisms has decreased from approximately 2 to 50 fold in the course of these passages.

The results of tests designed to detect a similar phenomenon for Rickettsia prowazekii have so far been negative. One series has been carried through 13 passages, and a second through six passages in chick embryos treated with partially inhibiting doses of chloramphenicol. Comparative tests of the passaged and stock strains have indicated no change in susceptibility to the drug under these conditions.

\section{SUMMARY AND CONCLUSIONS}

Sixty-four genera of micro-organisms including 290 species and strains have been tested for their susceptibility to chloramphenicol. The results are recorded in Tables I to IV.

From Table I it appears that all of the Rickettsiae tested are susceptible to chloramphenicol. Coxiella burnetii ( $Q$ Fever) may be more resistant than the others as larger doses are required to produce corresponding delay in mean death time of treated embryos. Possibly the whole lymphogranuloma venereum-psittacosis group of virus (Miyagawanella) will prove to be as susceptible as the type species. There are indications that influenza virus in mice and the virus of Newcastle disease of chickens respond somewhat to comparatively massive doses of the drug. It is possible, however, that the therapeutic effect seen can be explained by the action of the drug upon secondary bacterial invaders. While this hypothesis is apparently borne out by the negative results with chloramphenicol on these viruses in embryonated eggs, further work and possibly controlled clinical trials are indicated.

Tests against the virus of poliomyelitis have proved negative. Dr. J. L. Melnick states as follows: "I have gone over the records of the work done with Dr. Filiberto Ramirez Corria on the use of this material on two strains (Lansing and Y-Sk) of poliomyelitis virus and on Theiler's intestinal virus of mice. When the viruses were titrated using saturated aqueous solutions of Chloromycetin as diluent, there was no effect on the titer even when the viruses were inoculated into mice receiving daily inoculations of the drug ( $1 \mathrm{cc}$. of saturated solution intraperitoneally). Further- 
more, daily oral administration of Chloromycetin did not appear to have any effect on the intestinal carrier state of mice spontaneously harboring Theiler's virus of mouse encephalomyelitis" (22).

From Table II, which gives the results from tests of over 200 bacterial strains representing 25 genera, it can be concluded that considerable variation in susceptibility is encountered between genera as well as between species and even strains within the species. Under the conditions tested susceptibility to concentrations of $10 \mu \mathrm{g} / \mathrm{ml}$. or less, indicating possible clinical application, was observed for 21 of the genera. These include Aerobacter (2/2)," Bacillus (6/6), Brucella $(7 / 7)$, Corynebacterium (3/3), Diplococcus (3/3), Escherichia (11/11), Hemophilus (7/7), Klebsiella (11/12), Micrococcus (19/19), Neisseria (4/4), Pasteurella (8/8), Proteus (8/10), Pseudomonas (1/19), Salmonella (37/37), Sarcina (3/3), Shigella (16/16), Streptococcus $(8 / 8)$ and Vibrio (1/1). Pseudomonas, though fairly resistant, is included since it seems to be susceptible to chloramphenicol in concentrations obtainable in the urine. The results given for Alcaligenes leave the status of this genus somewhat in doubt. A stock strain of $A$. faecalis was found to be susceptible but three different isolates from the same clinical case of $A$. metalcaligenes infection were found to be highly resistant. A somewhat similar situation is observed for Proteus vulgaris where two of ten strains tested required a somewhat higher concentration ( $25 \mu \mathrm{g} / \mathrm{ml}$.) for complete inhibition. An interesting finding was encountered with the Clostridia. While this group in general was of very low susceptibility, one strain, the Mueller variant of $\mathrm{Cl}$. tetani, was very susceptible. This variant does not produce spores in culture, which raises the speculation that possibly the apparent resistance of this group is a reflection of the resistance of the spores rather than of the vegetative forms.

While not within the scope of this paper, in vivo laboratory trials with certain of the bacterial agents have been conducted. Gould et al. (26) report encouraging results with the cholera vibrio in mice and Gruhzit (25) has found activity in mice

\footnotetext{
- Indicates number susceptible strains/number of strains tested.
}

against certain of the Gram-positive cocci and the salmonella group. Sarber (27) has treated mice infected with Hemophilus pertussis and found chloramphenicol to be an effective chemotherapeutic agent. Clinical trials are in progress against many of these pathogens. To date clinical confirmation has been obtained in typhoid fever (19), brucellosis (20), and certain urinary infections caused by Gram-negative species (21).

Of the forty species or strains of fungi tested for susceptibility to chloramphenicol, Table III, only two were completely inhibited by the drug at the concentrations used in the test. These two species, Actinomyces bovis and Nocardia asteroides, both of which belong to the actinomycetes, were inhibited by $5 \mu \mathrm{g} / \mathrm{ml}$. and $20 \mu \mathrm{g} / \mathrm{ml}$. of the drug, respectively. Since blood levels of this magnitude are easily attained, trial on clinical infections with these agents is indicated.

Growth of four species of ringworm fungi, Trichophyton mentagrophytes, $T$. interdigitale, $T$. rubrum and Microsporum canis, was retarded by $1000 \mu \mathrm{g} / \mathrm{ml}$. of the drug but complete inhibition could not be obtained even when the drug concentration was increased to $2500 \mu \mathrm{g} / \mathrm{ml}$.

With the possible exception of the spirochete of relapsing fever (Borrelia recurrentis), the results with the spirochetes and protozoa tested were not promising. Doses of $12.5 \mathrm{mg} / \mathrm{Kg}$. twice daily for eight days were ineffective on syphilitic lesions in rabbits. Although similar treatment with 25 or $50 \mathrm{mg} / \mathrm{Kg}$. cleared the lesions, relapses occurred when treatment was stopped.

Attempts to induce increased resistance to chloramphenicol by passage in the presence of the drug have been successful in the case of seven bacterial species, but not for $R$. prowazekii in the embryonated egg. It remains to be seen whether, under practical conditions in the clinic, this finding will be of importance.

\section{BIBLIOGRAPHY}

1. Ehrlich, J., Bartz, Q. R., Smith, R. M., Joslyn, D. A., and Burkholder, P. R., Chloromycetin, a new antibiotic from a soil actinomycete. Science, 1947, 106, 417.

2. Ehrlich, J., Gottlieb, D., Burkholder, P. R., Anderson, L. E., and Pridham, T. G., Streptomyces venezuelae $n$. sp. the source of Chloromycetin. J. Bact., 1948, 56, 467. 
3. Carter, H. E., Gottlieb, D., and Anderson, H. W. Chloromycetin and streptothricin. Science, 1948, 107, 113.

4. Gottlieb, D., Bhattacharyya, P. K., Anderson, H. W., and Carter, H. E., Some properties of an antibiotic obtained from a species of Streptomyces. J. Bact., 1948, 55, 409.

5. Bartz, Q. R., Isolation and characterization of Chloromycetin. J. Biol. Chem., 1948, 172, 445.

6. Bartz, Q. R., Isolation and chemistry of chloramphenicol (Chloromycetin). J. Clin. Invest., 1949, 28, 1051.

7. Controulis, J., Rebstock, M. C., and Crooks, H. M., Jr., Chloramphenicol (Chloromycetin) v. synthesis. J. Am. Chem. Soc. In press.

8. Smadel, J. E., and Jackson, E. B., Chloromycetin, an antibiotic with chemotherapeutic activity in experimental rickettsial and viral infections. Science, 1947, 106, 418.

9. Smadel, J. E., and Jackson, E. B., Effect of Chloromycetin on experimental infection with psittacosis and lymphogranuloma venereum viruses. Proc. Soc. Exp. Biol. \& Med., 1948, 67, 478.

10. Smadel, J. E., Jackson, E. B., Ley, H. L., Jr., and Lewthwaite, R., Comparison of synthetic and fermentation chloramphenicol (Chloromycetin) in rickettsial and viral infections. Proc. Soc. Exp. Biol. \& Med., 1949, 70, 191.

11. Smadel, J. E., Jackson, E. B., and Cruise, A. B., Chloromycetin in experimental rickettsial infections. J. Immunol. In press.

12. Smith, R. M., Joslyn, D. A., Gruhzit, O. M., McLean, I. W., Jr., Penner, M. A., and Ehrlich, J., Chloromycetin : biological studies. J. Bact., 1948, 55, 425.

13. Youmans, G. P., Youmans, A. S., and Osborne, R. R., Tuberculostatic action of Chloromycetin in vitro and in vivo. Proc. Soc. Exp. Biol. \& Med., 1948, 67, 426.

14. Payne, E. H., Knaudt, J. A., and Palacios, S., Treatment of epidemic typhus with Chloromycetin. J. Trop. Med. \& Hyg., 1948, 51, 68.
15. Smadel, J. E., Woodward, T. E., Ley, H. L., Jr., Philip, C. B., Traub, R., Lewthwaite, R., and Savoor, S. R., Chloromycetin in the treatment of scrub typhus. Science, 1948, 108, 160.

16. Smadel, J. E., Leon, A. P., Ley, H. L., Jr., and Varela, G., Chloromycetin in the treatment of patients with typhus fever. Proc. Soc. Exp. Biol. \& Med., 1948, 68, 12.

17. Payne, E. H., Sharp, E. A., and Knaudt, J. A., Treatment of epidemic typhus with Chloromycetin. Trans. Royal Soc. Trop. Med. Hyg., 1948, 42, 163.

18. Pincoffs, M. C., Guy, E. G., Lister, L. M., Woodward, T. E., and Smadel, J. E., The treatment of Rocky Mountain spotted fever with Chloromycetin. Ann. Int. Med., 1948, 29, 656.

19. Woodward, T. E., Smadel, J. E., Ley, H. E., Jr., Green, R., and Mankikar, D. S., Preliminary report on the beneficial effect of Chloromycetin in the treatment of typhoid fever. Ann. Int. Med., 1948, 29, 131.

20. Woodward, T. E., Smadel, J. E., Holbrook, W. A., and Raby, W. R., The beneficial effect of Chloromycetin in brucellosis. J. Clin. Invest., 1949, 28, 968.

21. Chittenden, G. E., Sharp, E. A., Glazko, A. J., and Schlingman, A. S., Chloromycetin in therapy of bacillary urinary infection. J. Clin. Invest., 1949, 28, 1052.

22. Melnick, J. L. Personal communication.

23. Records, E., and Vawter, L. R., Tech. Bull. 173, Univ. Nev. Agr. Exp. Sta. (June) 1945.

24. Thompson, P. E. Personal communication.

25. Gruhzit, O. M. Personal communication.

26. Gould, R. L., Schlingman, A. S., Jackson, E. B., Manning, M. C., Batson, H. C., and Campbell, C. C., Chloramphenicol (Chloromycetin) in experimental cholera infections. J. Bact. In press.

27. Sarber, R. W., and Hemans, M. J., Chloramphenicol (Chloromycetin) in the chemotherapy of Hemophilus Pertussis infection in mice. Presented at 49th General Meeting Soc. American Bact., 1949, Cincinnati, Ohio. 